\title{
Hybrid Geometric Reduction of Hybrid Systems
}

\author{
Aaron D. Ames and Shankar Sastry
}

\begin{abstract}
This paper presents a unifying framework in which to carry out the hybrid geometric reduction of hybrid systems, generalizing classical reduction to a hybrid setting.
\end{abstract}

\section{INTRODUCTION}

The reduction of mechanical systems with symmetries plays a fundamental role in understanding the many important and interesting properties of these systems. Given a Hamiltonian on a symplectic manifold (the phase space) on which a Lie group acts symplectically, the main geometric reduction theorem [10] states that under certain conditions one can reduce the phase space to another symplectic manifold by "dividing out" by the symmetries. In addition, trajectories of the Hamiltonian on the phase space determine corresponding trajectories on the reduced space.

The goal of this paper is to generalize this result to a hybrid setting - $a$ formidable obstacle to which is the copious mathematical framework needed to perform reduction. Such a generalization of reduction, therefore, requires a new method for viewing "hybrid objects," one that allows classical mathematical objects and morphisms between these objects to be easily "hybridized." The framework in which we propose to carry out this generalization is that of category theory, and specifically through the notion of a hybrid object over a category (cf. [2], [3], [4]).

The objects of study in geometry display the fundamental property of being categorical, i.e., they reside in certain categories. Collections of geometric objects in a category relate to one another naturally, i.e., morphisms between geometric objects in two diagrams extend naturally to morphisms between diagrams. The relationship between different classes of geometric objects is functorial, i.e. one can translate from one class of geometric objects to another through the use of functors. Therefore, using the categorical, natural and functorial nature of geometric objects, one can hybridize these objects. Specifically, given a category $C$ consisting of the geometric objects of interest, e.g., manifolds, Lie groups, Lie algebras, etc., one can form the "hybrid version" of these geometric objects by considering hybrid objects over C consisting of pairs $(\mathcal{D}, \mathbf{A})$ where $\mathcal{D}$ is a small category of a specific form, termed a D-category, which encodes the discrete structure of the hybrid object and $\mathbf{A}: \mathcal{D} \rightarrow \mathrm{C}$ is a functor encoding its continuous structure.

This research is supported by the National Science Foundation (NSF award number CCR-0225610).

A. D. Ames is with the Control and Dynamical Systems Department, California Institute of Technology, Pasadena CA, 91125 amesecds.caltech.edu

S. Sastry is with the Department of Electrical Engineering and Computer Sciences, University of California at Berkeley, Berkeley CA, 94709 sastry@eecs.berkeley.edu
Utilizing hybrid objects over a general category, all of the major ingredients necessary for classical reduction can be hybridized. By leveraging the results of [10], we are able to show that when there is a hybrid symplectic manifold (the hybrid phase space) on which a hybrid Lie group acts symplectically, we can reduce the hybrid phase space to another hybrid symplectic manifold in which the hybrid symmetries are "divided out." In addition, hybrid trajectories of a hybrid Hamiltonian on the hybrid phase space determine corresponding hybrid trajectories on the reduced hybrid space. Thus, we generalize geometric reduction to a hybrid setting.

This paper draws heavily from two well-established areas of mathematics: classical mechanics (cf. [1], [6], [8], [9], [10]) and category theory (cf. [5]). All terms that are not defined in this paper can be found in these references. Much to the authors' regret, space constraints prevent us from including proofs and examples; these can be found in [2] (specifically, Chapters 1,2 and 4) along with detailed explanations of the concepts involved.

\section{HYBRID OBJECTS}

Hybrid objects provide a general framework in which to consider hybrid phenomena. This section introduces the foundations of this theory.

D-categories. Fundamental to the study of hybrid objects is the notion of $\mathrm{D}$-categories. These categories define the "discrete" structure of a hybrid object-the " $D$ " stands for discrete-and dictate how the "continuous components" of a hybrid object interact.

Let $\mathcal{D}$ be a small category with $\mathrm{Ob}(\mathcal{D})$ the set of objects of $\mathcal{D}$ and $\operatorname{Hom}_{\mathcal{D}}(a, b)$ the set of morphisms $\alpha: a \rightarrow b$. We use $\operatorname{Mor}(\mathcal{D})$ to denote the morphisms of $\mathcal{D}$, i.e.,

$$
\operatorname{Mor}(\mathcal{D})=\bigcup_{(a, b) \in \operatorname{Ob}(\mathcal{D}) \times \mathrm{Ob}(\mathcal{D})} \operatorname{Hom}_{\mathcal{D}}(a, b),
$$

and $\operatorname{Mor}_{\text {id }}(\mathcal{D})$ to denote the set of non-identity morphisms of $\mathcal{D}$, i.e.,

$$
\operatorname{Mor}_{\text {id }}(\mathcal{D})=\{\alpha \in \operatorname{Mor}(\mathcal{D}): \alpha \neq \mathrm{id}\} .
$$

For a morphism $\alpha: a \rightarrow b$ in $\mathcal{D}$, its domain is denoted by $\operatorname{dom}(\alpha)=a$ and its codomain is denoted by $\operatorname{cod}(\alpha)=b$.

Definition 2.1: A D-category is a small category $\mathcal{D}$ such that:

- There exist two subsets of $\mathrm{Ob}(\mathcal{D}), \mathrm{E}(\mathcal{D})$ and $\mathrm{V}(\mathcal{D})$, termed the edge set and vertex set, satisfying:

$$
\begin{aligned}
& \mathrm{E}(\mathcal{D}) \cap \mathrm{V}(\mathcal{D})=\emptyset, \\
& \mathrm{E}(\mathcal{D}) \cup \mathrm{V}(\mathcal{D})=\mathrm{Ob}(\mathcal{D}) .
\end{aligned}
$$


- There exists a pair of functions:

$$
\mathrm{E}(\mathcal{D}) \stackrel{\mathrm{s}}{\longrightarrow} \operatorname{Mor}_{\mathrm{id}}(\mathcal{D}),
$$

such that:

$$
\begin{aligned}
& \mathrm{s}(\mathrm{E}(\mathcal{D})) \cap \mathrm{t}(\mathrm{E}(\mathcal{D}))=\emptyset \\
& \mathrm{s}(\mathrm{E}(\mathcal{D})) \cup \mathrm{t}(\mathrm{E}(\mathcal{D}))=\operatorname{Mor}_{\text {id }}(\mathcal{D}) .
\end{aligned}
$$

The pair $(\mathrm{s}, \mathrm{t})$ is termed an orientation of $\mathcal{D}$.

- The following diagram:

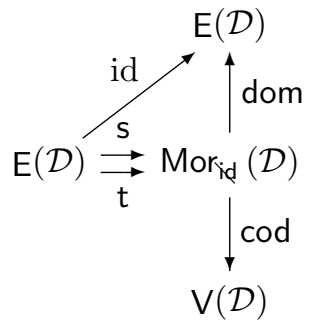

commutes.

The definition of a D-category implies that, for every $a \in$ $\mathrm{E}(\mathcal{D})$, there is a diagram in $\mathcal{D}$ :

$$
b \stackrel{\mathrm{s}_{a}}{\stackrel{\mathrm{t}_{a}}{\longrightarrow}} \mathrm{c}
$$

where $b, c \in \mathrm{V}(\mathcal{D})$. Diagrams of this form can be thought of as the "canonical" D-categories.

D-categories and graphs. Define the category of Dcategories, Dcat, to have as objects D-categories. A morphism between two D-categories, $\mathcal{D}$ and $\mathcal{D}^{\prime}$ (with orientations $(\mathrm{s}, \mathrm{t})$ and $\left(\mathrm{s}^{\prime}, \mathrm{t}^{\prime}\right)$, respectively), is a functor $\vec{F}: \mathcal{D} \rightarrow \mathcal{D}^{\prime}$ such that

$$
\vec{F}(\mathrm{~V}(\mathcal{D})) \subseteq \vec{F}\left(\mathrm{~V}\left(\mathcal{D}^{\prime}\right)\right), \quad \vec{F}(\mathrm{E}(\mathcal{D})) \subseteq \vec{F}\left(\mathrm{E}\left(\mathcal{D}^{\prime}\right)\right)
$$

and

$$
\vec{F}\left(\mathrm{~s}_{a}\right)=\mathrm{s}_{\vec{F}(a)}^{\prime}, \quad \vec{F}\left(\mathrm{t}_{a}\right)=\mathrm{t}_{\vec{F}(a)}^{\prime},
$$

for every $a \in \mathrm{E}(\mathcal{D})$. That is, a morphism of D-categories is a functor that preserves vertex sets, edge sets and orientations.

To every D-category, there is an associated oriented graph and, conversely, to every oriented graph there is an associated D-category. In fact, there is an isomorphism of categories: Dcat $\cong$ Grph, with Grph the category of oriented graphs. For a detailed discussion on D-categories, including numerous examples, we refer the reader to [2].

With the notion of a D-category in hand, we can define hybrid objects over a category $C$. Note that from this point on, we will denote $\mathrm{D}$-categories by calligraphic symbols: $\mathcal{A}$, $\mathcal{B}, \mathcal{C}, \mathcal{D}$, et cetera.

Definition 2.2: Let $\mathrm{C}$ be a category. A hybrid object over $\mathrm{C}$ is a pair $(\mathcal{A}, \mathbf{A})$, where $\mathcal{A}$ is a D-category and

$$
\mathbf{A}: \mathcal{A} \rightarrow \mathrm{C}
$$

is a covariant functor. A cohybrid object over $\mathrm{C}$ is a pair $(\mathcal{A}, \mathbf{A})$ where $\mathbf{A}: \mathcal{A} \rightarrow \mathrm{C}$ is a contravariant functor.

We are interested in considering the category of all hybrid (cohybrid) objects over a category C. First, some notation is needed. Let $\mathrm{C}^{\mathcal{A}}$ denote the category with objects covariant functors $\mathbf{A}: \mathcal{A} \rightarrow \mathrm{C}$ and morphisms natural transformations. Similarly, let ${ }^{\mathcal{B}} \mathrm{C}$ denote the category of contravariant functors $\mathbf{B}: \mathcal{B} \rightarrow \mathrm{C}$. Finally, $\mathrm{C}^{\text {op }}$ denotes the opposite category to $\mathrm{C}$ given by "reversing" the morphisms.

Definition 2.3: Let $\mathrm{C}$ be a category. The category of hybrid objects over the category $\mathrm{C}$, denoted by $\mathrm{Hy}(\mathrm{C})$, has as

Objects: Hybrid objects over $\mathrm{C}$.

Morphisms: Pairs $(\vec{F}, \vec{f}):(\mathcal{A}, \mathbf{A}) \rightarrow(\mathcal{B}, \mathbf{B})$ where $\vec{F}$ is a morphism in Dcat and $\vec{f}: \mathbf{A} \dot{\rightarrow} \circ \vec{F}$ is a morphism in $C^{\mathcal{A}}$.

The category of cohybrid objects over the category C, denoted by $\mathrm{CoHy}(\mathrm{C})$, has as

Objects: Cohybrid objects over $\mathrm{C}$. Morphisms: Pairs $\left(\vec{F}^{\circ \mathrm{op}}, \vec{f}\right):(\mathcal{A}, \mathbf{A}) \rightarrow(\mathcal{B}, \mathbf{B})$ where $\vec{F}^{\text {op }}: \mathcal{A} \rightarrow \mathcal{B}$ is the morphism in Dcat ${ }^{\text {op }}$ corresponding to the morphism $\vec{F}: \mathcal{B} \rightarrow \mathcal{A}$ in Dcat and $\vec{f}: \mathbf{A} \circ \vec{F} \dot{\rightarrow} \mathbf{B}$ in ${ }^{\mathcal{B}} \mathrm{C}$.

Note 2.1: We often will be interested in only a single hybrid object and its relation to hybrid objects with the same D-category, i.e., hybrid objects with the same discrete structure. In this case, we will denote such a hybrid object by $\mathbf{A}$ and a morphism between it and another hybrid object, $\mathbf{A}^{\prime}$, by $\vec{f}$; that is, $\mathbf{A}$ represents the hybrid object $(\mathcal{A}, \mathbf{A})$, $\mathbf{A}^{\prime}$ represents the hybrid object $\left(\mathcal{A}, \mathbf{A}^{\prime}\right)$ and $\vec{f}$ represents the hybrid morphism $\left(\overrightarrow{\mathrm{Id}}_{\mathcal{A}}, \vec{f}\right)$, where $\overrightarrow{\mathrm{Id}}_{\mathcal{A}}$ is the identity functor.

Elements of hybrid and cohybrid objects. Central to many of the constructions that will be introduced is the notion of an element of a hybrid (cohybrid) object. To discuss elements, we suppose that $C$ is a category such that there is a forgetful functor $U: C \rightarrow$ Set with Set the category of sets, i.e., the objects of $C$ have the underlying structure of a set.

An element of a hybrid object $(\mathcal{A}, \mathbf{A})$, denoted by $\vec{e} \in$ $(\mathcal{A}, \mathbf{A})$ (or just $\vec{e} \in \mathbf{A}$ when the D-category is clear from context), is a set $\vec{e}=\left\{\vec{e}_{a}\right\}_{a \in \mathrm{Ob}(\mathcal{A})}$ that must satisfy the following properties:

- $\vec{e}_{a} \in \mathbf{A}_{a}$ for all $a \in \mathrm{Ob}(\mathcal{A})$,

- $\vec{e}_{b}=\mathbf{A}_{\alpha}\left(\vec{e}_{a}\right)$ for all $\alpha \in \operatorname{Hom}_{\mathcal{A}}(a, b)$.

Similarly, an element of a cohybrid object $(\mathcal{A}, \mathbf{A})$, denoted by $\vec{\omega} \in(\mathcal{A}, \mathbf{A})$, is a set $\vec{\omega}=\left\{\vec{\omega}_{a}\right\}_{a \in \mathrm{Ob}(\mathcal{A})}$ that must satisfy the following properties:

- $\vec{\omega}_{a} \in \mathbf{A}_{a}$ for all $a \in \mathrm{Ob}(\mathcal{A})$,

- $\mathbf{A}_{\alpha}\left(\vec{\omega}_{b}\right)=\vec{\omega}_{a}$ for all $\alpha \in \operatorname{Hom}_{\mathcal{A}}(a, b)$.

Note that for $\vec{f}: \mathbf{A} \dot{\rightarrow} \mathbf{A}^{\prime}$, if $\vec{\omega} \in(\mathcal{A}, \mathbf{A})$ then $\vec{f}(\vec{\omega})=$ $\left\{\vec{f}_{a}\left(\vec{\omega}_{a}\right)\right\}_{a \in \mathrm{Ob}(\mathcal{A})} \in\left(\mathcal{A}, \mathbf{A}^{\prime}\right)$.

Functors between categories of hybrid objects. Consider a covariant functor $G: \mathrm{C} \rightarrow \mathrm{D}$ between two categories. This functor induces a functor, termed the pushforward, $G_{*}$ : $\mathrm{C}^{\mathcal{D}} \rightarrow \mathrm{D}^{\mathcal{D}}$ between functor categories, i.e., $G_{*}(\mathbf{A})=G \circ \mathbf{A}$. This functor, in turn, induces a functor:

$$
\mathrm{Hy}(G): \mathrm{Hy}(C) \rightarrow \mathrm{Hy}(\mathrm{D})
$$


between categories of hybrid objects. On objects $(\mathcal{A}, \mathbf{A})$ and morphisms $(\vec{F}, \vec{f}):(\mathcal{A}, \mathbf{A}) \rightarrow(\mathcal{B}, \mathbf{B})$ of $\mathrm{Hy}(\mathbf{C})$, the functor $\mathrm{Hy}(G)$ is given by:

$$
\begin{aligned}
\operatorname{Hy}(G)(\mathcal{A}, \mathbf{A}) & :=\left(\mathcal{A}, G_{*}(\mathbf{A})\right), \\
\operatorname{Hy}(G)(\vec{F}, \vec{f}) & :=\left(\vec{F}, G_{*}(\vec{f})\right) .
\end{aligned}
$$

If $G: \mathrm{C} \rightarrow \mathrm{D}$ is a contravariant functor then its pushfoward is a functor $G_{*}: \mathrm{C}^{\mathcal{A}} \rightarrow{ }^{\mathcal{A}} \mathrm{D}$, which thus induces a contravariant functor

$$
\mathrm{Hy}(G): \mathrm{Hy}(\mathrm{C}) \rightarrow \mathrm{CoHy}(\mathrm{D})
$$

given on objects and morphisms of $\mathrm{Hy}(\mathrm{C})$ by:

$$
\begin{aligned}
\operatorname{Hy}(G)(\mathcal{A}, \mathbf{A}) & :=\left(\mathcal{A}, G_{*}(\mathbf{A})\right), \\
\operatorname{Hy}(G)(\vec{F}, \vec{f}) & :=\left(\vec{F}^{\mathrm{op}}, G_{*}(\vec{f})\right),
\end{aligned}
$$

where $\vec{F}^{\mathrm{op}}: \mathcal{B} \rightarrow \mathcal{A}$ is the morphism in Dcat ${ }^{\mathrm{op}}$ corresponding to the morphism $\vec{F}: \mathcal{A} \rightarrow \mathcal{B}$ in Dcat.

\section{HybRID SyMPLECTIC MANIFOLDS}

To illustrate the descriptive power of hybrid objects, we introduce hybrid symplectic manifolds utilizing this framework.

Hybrid tangent bundles. We begin by discussing how one associates to a hybrid manifold its hybrid tangent bundle. This will be useful for understanding later constructions.

The process of associating a tangent bundle to a manifold defines a functor:

$$
T: \text { Man } \rightarrow \text { VectBund }_{\mathbb{R}},
$$

where Man is the category of manifolds (the objects of this category are smooth manifolds and the morphisms are smooth maps between manifolds) and VectBund $\mathbb{R}_{\mathbb{R}}$ is the category of (real) vector bundles. Specifically, we have $T(M)=$ $T M$, where $T M$ is the tangent bundle of $M$; implicit in this notation is the canonical projection $\pi: T M \rightarrow M$ that makes $T M$ into a vector bundle. In addition, for a morphism $f$ of manifolds $T f$ is the pushfoward of this function.

The functor $T$ induces a functor:

$$
\mathrm{Hy}(T): \mathrm{Hy}(\text { Man }) \rightarrow \mathrm{Hy}\left(\text { VectBund }_{\mathbb{R}}\right) .
$$

That is, for a hybrid manifold $(\mathcal{M}, \mathbf{M})$, i.e., $\mathbf{M}: \mathcal{M} \rightarrow$ Man, we can associate to this hybrid manifold its hybrid tangent bundle

$$
\left(\mathcal{M}, T_{*}(\mathbf{M})\right)=\mathrm{Hy}(T)(\mathcal{M}, \mathbf{M}) .
$$

Hybrid differential forms. Let $M$ be a manifold and let $\Omega^{k}(M)$ be the vector space of differential k-forms. The process of associating to a manifold its differential k-forms induces a contravariant functor:

$$
\Omega^{k}: \operatorname{Man} \rightarrow \operatorname{Vect}_{\mathbb{R}},
$$

where for $f: M \rightarrow N, \Omega^{k}(f): \Omega^{k}(N) \rightarrow \Omega^{k}(M)$ is the pullback of $f$.

The contravariant functor $\Omega^{k}$ induces a contravariant functor:

$$
\mathrm{Hy}\left(\Omega^{k}\right): \mathrm{Hy}(\mathrm{Man}) \rightarrow \mathrm{CoHy}\left(\operatorname{Vect}_{\mathbb{R}}\right),
$$

where, for a hybrid manifold $(\mathcal{M}, \mathbf{M})$,

$$
\mathrm{Hy}\left(\Omega^{k}\right)(\mathcal{M}, \mathbf{M})=\left(\mathcal{M}, \Omega_{*}^{k}(\mathbf{M})\right),
$$

with $\Omega_{*}^{k}(\mathbf{M}): \mathcal{M} \rightarrow \operatorname{Vect}_{\mathbb{R}}$ a contravariant functor.

We are especially interested in elements of the cohybrid object $\left(\mathcal{M}, \Omega_{*}^{k}(\mathbf{M})\right)$.

Definition 3.1: Let $(\mathcal{M}, \mathbf{M})$ be a hybrid manifold. A hybrid differential $k$-form, $\vec{\omega}$, is an element of the cohybrid object $\left(\mathcal{M}, \Omega_{*}^{k}(\mathbf{M})\right)$. Therefore, a hybrid differential k-form must satisfy:

- $\vec{\omega}_{a} \in \Omega^{k}\left(\mathbf{M}_{a}\right)$, i.e, $\vec{\omega}_{a}$ is a differential k-form, for all $a \in \mathrm{Ob}(\mathcal{M})$,

- $\Omega^{k}\left(\mathbf{M}_{\alpha}\right)\left(\vec{\omega}_{b}\right)=\vec{\omega}_{a}$ for all $\alpha \in \operatorname{Hom}_{\mathcal{M}}(a, b)$.

Hybrid symplectic manifolds. The formulation of hybrid differential forms allows us to define hybrid symplectic manifolds. Note that the definition of a hybrid symplectic manifold is not the most obvious one-we do not require a hybrid differential 2-form to be objectwise a symplectic form.

Definition 3.2: A hybrid symplectic manifold is a hybrid manifold $(\mathcal{M}, \mathbf{M})$ together with a hybrid 2-form $\vec{\omega} \in \Omega_{*}^{2}(\mathbf{M})$ such that $\vec{\omega}_{b}$ is a symplectic form for all $b \in \mathrm{V}(\mathcal{M})$.

\section{Hybrid Lie GROUPS AND Algebras}

Symmetries lie at the heart of classical reduction. This also will be the case with hybrid reduction, so we must necessarily consider hybrid symmetries. These take the form of hybrid Lie groups, which we introduce in this section along with the corresponding notion of a hybrid Lie algebra.

Hybrid Lie groups. A hybrid Lie group is a hybrid object over the category of Lie groups, LieGrp, i.e., a pair $(\mathcal{G}, \mathbf{G})$ where

$$
\mathbf{G}: \mathcal{G} \rightarrow \text { LieGrp. }
$$

An element of a hybrid Lie group, $\vec{g} \in \mathbf{G}$, must satisfy the following properties:

- $\vec{g}_{a} \in \mathbf{G}_{a}$ for all $a \in \mathrm{Ob}(\mathcal{G})$,

- $\vec{g}_{b}=\mathbf{G}_{\alpha}\left(\vec{g}_{a}\right)$ for all $\alpha \in \operatorname{Hom}_{\mathcal{G}}(a, b)$.

In particular, every element of $(\mathcal{G}, \mathbf{G})$ has an inverse, $\vec{g}^{-1}$, defined objectwise to be the inverse of $\vec{g}_{a}$.

The Lie functor. The Lie functor is the functor:

$$
\text { Lie }: \text { LieGrp } \rightarrow \text { LieAlg, }
$$

with LieAlg the category of Lie algebras. This functor is given on objects by associating to a Lie group $G$ its Lie algebra:

$$
\operatorname{Lie}(G)=T_{e_{G}} G,
$$

where $T_{e_{G}} G$ is the tangent space at the identity element of $G$. For a morphism $f: G \rightarrow H$ of Lie groups,

$$
\operatorname{Lie}(f):=T_{e_{G}} f: \operatorname{Lie}(G) \rightarrow \operatorname{Lie}(H) .
$$

Note that $\operatorname{Lie}(G)$ is typically denoted by $\mathfrak{g}$.

The Lie functor yields a functor between categories of hybrid objects:

$$
\mathrm{Hy}(\mathrm{Lie}): \mathrm{Hy}(\text { LieGrp }) \rightarrow \mathrm{Hy}(\text { LieAlg }) .
$$


For a hybrid Lie group $(\mathcal{G}, \mathbf{G})$, we will denote its corresponding hybrid Lie algebra by

$$
(\mathcal{G}, \mathfrak{g}):=\operatorname{Hy}(\operatorname{Lie})(\mathcal{G}, \mathbf{G}),
$$

where $\mathfrak{g}: \mathcal{G} \rightarrow$ LieAlg. We know that a hybrid Lie algebra is also a hybrid vector space, $\mathfrak{g}: \mathcal{G} \rightarrow \operatorname{Vect}_{\mathbb{R}}$, since the category of Lie algebras is a subcategory of the category of vector spaces. Therefore, an element of $(\mathcal{G}, \mathfrak{g})$, which we denote by $\vec{\xi} \in(\mathcal{G}, \mathfrak{g})$ (or just $\vec{\xi} \in \mathfrak{g}$ ), is a hybrid vector and so must satisfy:

- $\vec{\xi}_{a} \in \mathfrak{g}_{a}$ for all $a \in \mathrm{Ob}(\mathcal{G})$, i.e., $\vec{\xi}_{a}$ is a vector,

- $\vec{\xi}_{b}=\mathfrak{g}_{\alpha}\left(\vec{\xi}_{a}\right)$ for all $\alpha \in \operatorname{Hom}_{\mathcal{G}}(a, b)$.

The dual to a hybrid Lie algebra. The contravariant functor that associates to a vector space its dual:

$$
(-)^{\star}: \operatorname{Vect}_{\mathbb{R}} \rightarrow \operatorname{Vect}_{\mathbb{R}},
$$

induces a functor between categories of hybrid and cohybrid objects:

$$
\mathrm{Hy}\left((-)^{\star}\right): \mathrm{Hy}\left(\operatorname{Vect}_{\mathbb{R}}\right) \rightarrow \mathrm{CoHy}\left(\operatorname{Vect}_{\mathbb{R}}\right) .
$$

Through this functor we obtain the dual to a hybrid Lie algebra $(\mathcal{G}, \mathfrak{g})$, which is the cohybrid object:

$$
\left(\mathcal{G}, \mathfrak{g}^{\star}\right):=\operatorname{Hy}\left((-)^{\star}\right)(\mathcal{G}, \mathfrak{g}) .
$$

An element of the cohybrid object $\left(\mathcal{G}, \mathfrak{g}^{\star}\right), \vec{\mu} \in\left(\mathcal{G}, \mathfrak{g}^{\star}\right)$ (or just $\left.\vec{\mu} \in \mathfrak{g}^{\star}\right)$, is a hybrid covector and thus must satisfy:

- $\vec{\mu}_{a} \in \mathfrak{g}_{a}^{\star}$ for all $a \in \mathrm{Ob}(\mathcal{G})$, i.e., $\vec{\mu}_{a}: \mathfrak{g}_{a} \rightarrow \mathbb{R}$ is a covector,

- $\mathfrak{g}_{\alpha}^{\star}\left(\vec{\mu}_{b}\right)=\vec{\mu}_{a}$ for all $\alpha \in \operatorname{Hom}_{\mathcal{G}}(a, b)$, i.e., $\vec{\mu}_{b} \circ \mathfrak{g}_{\alpha}=\vec{\mu}_{a}$. This implies that $\vec{\mu}$ corresponds to a natural transformation $\vec{\mu}: \mathbf{G} \dot{\rightarrow} \Delta_{\mathcal{G}}(\mathbb{R})$, with $\Delta_{\mathcal{G}}$ the constant functor.

The hybrid adjoint action. Let $G$ be a Lie group and $g \in$ $G$. The conjunction map is defined to be a map $I_{g}: G \rightarrow G$ with $I_{g}(h)=g h g^{-1}$ for $h \in G$. Utilizing the Lie functor, we obtain a Lie algebra homomorphism:

$$
\operatorname{Ad}_{g}:=\operatorname{Lie}\left(I_{g}\right): \mathfrak{g} \rightarrow \mathfrak{g},
$$

which is termed the adjoint action. Applying the functor $(-)^{\star}$ yields a morphism of vector spaces:

$$
\operatorname{Ad}_{g}^{\star}: \mathfrak{g}^{\star} \rightarrow \mathfrak{g}^{\star}
$$

termed the coadjoint action.

The framework of hybrid objects allows coadjoint actions to be easily generalized to a hybrid setting. Given an element $\vec{g} \in(\mathcal{G}, \mathbf{G})$, we obtain a morphism of hybrid objects:

$$
\left(\overrightarrow{\operatorname{Id}}_{\mathcal{G}}, \vec{I}_{\vec{g}}\right):(\mathcal{G}, \mathbf{G}) \rightarrow(\mathcal{G}, \mathbf{G}),
$$

with $\vec{I}_{\vec{g}}$ defined objectwise by $\left(\vec{I}_{\vec{g}}\right)_{a}=I_{\vec{g}_{a}}$. Utilizing the functors $\mathrm{Hy}(\mathrm{Lie})$ and $\mathrm{Hy}\left((-)^{\star}\right)$, we obtain the hybrid coadjoint action, i.e., the morphism of cohybrid objects:

$$
\left(\overrightarrow{\mathrm{Id}}_{\mathcal{G}}, \overrightarrow{\operatorname{Ad}}_{\vec{g}}^{\star}\right):=\operatorname{Hy}\left((-)^{\star}\right)\left(\mathrm{Hy}(\operatorname{Lie})\left(\left(\overrightarrow{\mathrm{Id}}_{\mathcal{G}}, \vec{I}_{\vec{g}}\right)\right)\right) .
$$

That is, $\overrightarrow{A d}_{\vec{g}}^{\star}: \mathfrak{g}^{\star} \dot{\rightarrow} \mathfrak{g}^{\star}$ and it follows that $\left(\overrightarrow{\mathrm{Ad}}_{\vec{g}}^{\star}\right)_{a}=\operatorname{Ad}_{\vec{g}_{a}}^{\star}$.
Hybrid isotropy subgroups. For a Lie group $G$, the isotropy subgroup under the coadjoint action is given by, for $\mu \in \mathfrak{g}^{\star}$,

$$
G_{\mu}=\left\{g \in G: \operatorname{Ad}_{g^{-1}}^{\star}(\mu)=\mu\right\} .
$$

For $\vec{\mu} \in\left(\mathcal{G}, \mathfrak{g}^{\star}\right)$, define the hybrid isotropy group as the hybrid Lie group

$$
\mathbf{G}_{\vec{\mu}}: \mathcal{G} \rightarrow \text { LieGrp }
$$

defined on objects and morphisms of $\mathcal{G}$ by $\left(\mathbf{G}_{\vec{\mu}}\right)_{a}=\left(\mathbf{G}_{a}\right)_{\vec{\mu}_{a}}$ and $\left(\mathbf{G}_{\vec{\mu}}\right)_{\alpha}=\left.\left(\mathbf{G}_{\alpha}\right)\right|_{\left(\mathbf{G}_{\vec{\mu}}\right)_{a}}$.

\section{HybRid Momentum MaPs}

Momentum maps make explicit the conserved quantities of a Hamiltonian system. Hybrid momentum maps serve the same function except that they define a set of conserved quantities. In order to introduce hybrid momentum maps, it is first necessary to introduce the notion of hybrid symmetries, i.e., a hybrid action of a hybrid Lie group on a hybrid manifold. When such hybrid symmetries exists, along with a momentum map, we are able to "divide out" by these symmetries to obtain the reduced hybrid phase space.

Hybrid group actions. For $\mathbf{G}: \mathcal{M} \rightarrow$ LieGrp and $\mathbf{M}$ : $\mathcal{M} \rightarrow$ Man, define the hybrid manifold $\mathbf{G} \times \mathbf{M}: \mathcal{M} \rightarrow$ Man as the product of $\mathbf{G}$ and $\mathbf{M}$ in $\mathbf{M a n}^{\mathcal{M}}$, i.e., on objects and morphisms:

$$
(\mathbf{G} \times \mathbf{M})_{a}:=\mathbf{G}_{a} \times \mathbf{M}_{a}, \quad(\mathbf{G} \times \mathbf{M})_{\alpha}:=\left(\mathbf{G}_{\alpha}, \mathbf{M}_{\alpha}\right) .
$$

A hybrid group action or hybrid action is a natural transformation

$$
\vec{\Phi}: \mathbf{G} \times \mathbf{M} \dot{\hookrightarrow} \mathbf{M}
$$

that is objectwise a group action:

- For all $p \in \mathbf{M}_{a}, \vec{\Phi}_{a}\left(e_{\mathbf{G}_{a}}, p\right)=p$,

- For every $g, h \in \mathbf{G}_{a}, \vec{\Phi}_{a}\left(g, \vec{\Phi}_{a}(h, p)\right)=\vec{\Phi}_{a}(g h, p)$.

We say that a hybrid group action is free if $\vec{\Phi}$ is objectwise free, and it is proper if it is objectwise proper.

For $\vec{g} \in \mathbf{G}$, we can associate to this action a hybrid diffeomorphism (a natural isomorphism)

$$
\vec{\Phi}_{\vec{g}}: \mathbf{M} \dot{\rightarrow} \mathbf{M}
$$

defined objectwise, for $p \in \mathbf{M}_{a}$, by $\left(\vec{\Phi}_{\vec{g}}\right)_{a}(p):=\vec{\Phi}_{a}\left(\vec{g}_{a}, p\right)$.

Definition 5.1: Let $(\mathbf{M}, \vec{\omega})$ be a hybrid symplectic manifold. A hybrid action $\vec{\Phi}: \mathbf{G} \times \mathbf{M} \rightarrow \mathbf{M}$ is a symplectic hybrid action if for the hybrid diffeomorphism $\vec{\Phi}_{\vec{g}}: \mathbf{M} \doteq \mathbf{M}$,

$$
\Omega_{*}^{2}\left(\vec{\Phi}_{\vec{g}}\right)(\vec{\omega})=\vec{\omega}
$$

for each $\vec{g} \in \mathbf{G}$.

Hybrid orbit spaces. For a hybrid manifold $\mathbf{M}$ with a hybrid group $\mathbf{G}$ acting on it, let $\mathbf{M}_{a} / \mathbf{G}_{a}$ be the orbit space of the action $\vec{\Phi}_{a}$ of $\mathbf{G}_{a}$ on $\mathbf{M}_{a}$; if $p \in \mathbf{M}_{a}$, we denote the elements of this space by $[p]$. Define the hybrid topological space

$$
\mathbf{M} / \mathbf{G}: \mathcal{M} \rightarrow \text { Top, }
$$


with Top the category of topological spaces, on objects and morphisms of $\mathcal{M}$ by $\mathbf{M} / \mathbf{G}_{a}:=\mathbf{M}_{a} / \mathbf{G}_{a}$ and $\mathbf{M} / \mathbf{G}_{\alpha}([p]):=\left[\mathbf{M}_{\alpha}(p)\right]$.

Proposition 5.1: If $\vec{\Phi}: \mathbf{G} \times \mathbf{M} \doteq \mathbf{M}$ is a free and proper hybrid action, then $\mathbf{M} / \mathbf{G}$ is a hybrid manifold, i.e., $\mathbf{M} / \mathbf{G}$ : $\mathcal{M} \rightarrow$ Man. Moreover, there is a hybrid submersion:

$$
\vec{\pi}: \mathbf{M} \dot{\rightarrow} \mathbf{M} \mathbf{G} .
$$

That is, $\vec{\pi}$ is a natural transformation that is objectwise a submersion.

Hybrid infinitesimal generators. Suppose there is a hybrid action $\vec{\Phi}: \mathbf{G} \times \mathbf{M} \dot{\rightarrow} \mathbf{M}$. Define the infinitesimal generator of the hybrid action $\vec{\Phi}$ corresponding to $\vec{\xi} \in \mathfrak{g}$ by

$$
\vec{\xi}_{\mathbf{M}}: \mathbf{M} \dot{\rightarrow} T_{*}(\mathbf{M})
$$

which is given objectwise by

$$
\left(\vec{\xi}_{\mathbf{M}}\right)_{a}(p):=\left.\frac{\mathrm{d}}{\mathrm{dt}} \vec{\Phi}_{a}\left(\exp \left(t \vec{\xi}_{a}\right), p\right)\right|_{t=0}
$$

for $p \in \mathbf{M}_{a}$.

Hybrid regular values. Consider a natural transformation $\vec{J}: \mathbf{M} \dot{\rightarrow} \mathfrak{g}^{\star} ;$ since $\mathbf{M}$ is covariant and $\mathfrak{g}^{\star}$ is contravariant, this implies that the following diagram

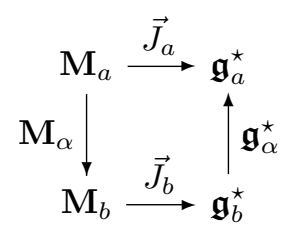

must commute for all $\alpha \in \operatorname{Hom}_{\mathcal{M}}(a, b)$.

Definition 5.2: We say that $\vec{\mu} \in \mathfrak{g}^{\star}$ is a hybrid regular value of $\vec{J}$ if

1) $\vec{\mu}_{a} \in \mathfrak{g}_{a}^{\star}$ is a regular value of $\vec{J}_{a}: \mathbf{M}_{a} \rightarrow \mathfrak{g}_{a}^{\star}$ for all $a \in \mathrm{Ob}(\mathcal{M})$

2) $\vec{\mu}_{b}=\vec{J}_{b} \circ \mathbf{M}_{\alpha}(p)$ for all $\alpha \in \operatorname{Hom}_{\mathcal{M}}(a, b)$ and $p \in \mathbf{M}_{a}$ such that $\vec{J}_{a}(p)=\vec{\mu}_{a}$.

The hybrid manifold $\mathbf{J}^{-1}(\vec{\mu})$. Given a hybrid regular value $\vec{\mu} \in \mathfrak{g}^{\star}$, define a hybrid manifold

$$
\mathbf{J}^{-1}(\vec{\mu}): \mathcal{M} \rightarrow \text { Man }
$$

given on objects and morphisms of $\mathcal{M}$ by $\mathbf{J}^{-1}(\vec{\mu})_{a}:=$ $\vec{J}_{a}^{-1}\left(\vec{\mu}_{a}\right)$ and $\mathbf{J}^{-\mathbf{1}}(\overrightarrow{\boldsymbol{\mu}})_{\alpha}:=\left.\mathbf{M}_{\alpha}\right|_{\mathbf{J}^{-1}(\vec{\mu})_{a}}$. Note that there is a hybrid inclusion (a natural transformation that is objectwise an inclusion),

$$
\vec{i}_{\vec{\mu}}: \mathbf{J}^{-1}(\vec{\mu}) \dot{M}
$$

Induced natural transformations. Given a natural transformation $\vec{J}: \mathbf{M} \dot{\rightarrow} \mathfrak{g}^{\star}$, for all $\vec{\xi} \in \mathfrak{g}$ we can define a natural transformation:

$$
\overrightarrow{J \xi}: \mathbf{M} \dot{\rightarrow} \Delta_{\mathcal{M}}(\mathbb{R})
$$

given objectwise by $\overrightarrow{J \xi}_{a}(p):=\left\langle\vec{J}_{a}(p), \vec{\xi}_{a}\right\rangle=\vec{J}_{a}(p)\left(\vec{\xi}_{a}\right)$, for $p \in \mathbf{M}_{a}$.
Definition 5.3: Let $(\mathbf{M}, \vec{\omega})$ be a hybrid symplectic manifold and let $\vec{\Phi}: \mathbf{G} \times \mathbf{M} \dot{\hookrightarrow} \mathbf{M}$ be a hybrid action. Define a hybrid momentum map as a natural transformation

$$
\vec{J}: \mathbf{M} \dot{\rightarrow} \mathfrak{g}^{\star}
$$

such that for every $\vec{\xi} \in \mathfrak{g}$,

$$
d\left(\vec{J}_{a}\right)=\iota_{\left(\vec{\xi}_{\mathrm{M}}\right)_{a}}\left(\vec{\omega}_{a}\right)
$$

for all $a \in \operatorname{Ob}(\mathcal{M})$, where $\vec{\xi}_{\mathrm{M}}$ is the hybrid infinitesimal generator of the hybrid action corresponding to $\vec{\xi}$.

Definition 5.4: Let $\vec{\Phi}: \mathbf{G} \times \mathbf{M} \doteq \mathbf{M}$ be a hybrid action of $\mathbf{G}$ on $\mathbf{M}$. A hybrid momentum map is said to be $\overrightarrow{\mathrm{Ad}}^{\star}$ equivariant under this action if for every $\vec{g} \in \mathbf{G}$ the following diagram of natural transformations commutes:

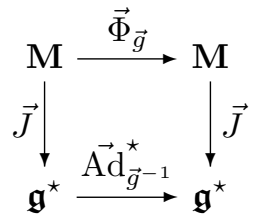

The reduced hybrid phase space. Suppose that $\mathbf{G}$ acts on $\mathbf{M}$ through the hybrid action $\vec{\Phi}$, and let $\vec{J}: \mathbf{M} \dot{\rightarrow} \mathfrak{g}^{\star}$ be an $\overrightarrow{\mathrm{Ad}}^{\star}$-equivariant hybrid momentum map. Assume that $\vec{\mu} \in \mathfrak{g}^{\star}$ is a hybrid regular value of $\vec{J}$; therefore, $\mathbf{J}^{-\mathbf{1}}(\overrightarrow{\boldsymbol{\mu}})$ : $\mathcal{M} \rightarrow$ Man is a hybrid manifold. The restriction of the hybrid action $\vec{\Phi}_{\vec{g}}$ to $\mathbf{J}^{-1}(\vec{\mu})$ and $\mathbf{G}_{\vec{\mu}}$ (also denoted by $\vec{\Phi}_{\vec{g}}$ ):

$$
\vec{\Phi}_{\vec{g}}: \mathbf{J}^{-1}(\vec{\mu}) \dot{\rightarrow} \mathbf{J}^{-1}(\vec{\mu}), \quad \vec{g} \in \mathbf{G}_{\vec{\mu}},
$$

is a hybrid action. In other words, if $\mathbf{G}$ acts on $\mathbf{M}$, then $\mathbf{G}_{\vec{\mu}}$ acts on $\mathbf{J}^{-1}(\vec{\mu})$.

If $\mathbf{G}_{\vec{\mu}}$ acts freely and properly on $\mathbf{J}^{-1}(\vec{\mu})$, then

$$
\mathbf{M}_{\vec{\mu}}:=\mathbf{J}^{-1}(\vec{\mu}) / \mathbf{G}_{\vec{\mu}}: \mathcal{M} \rightarrow \text { Man }
$$

is a hybrid manifold and the canonical hybrid projection

$$
\vec{\pi}_{\vec{\mu}}: \mathbf{J}^{-1}(\vec{\mu}) \dot{\rightarrow} \mathbf{M}_{\vec{\mu}}=\mathbf{J}^{-1}(\vec{\mu}) / \mathbf{G}_{\vec{\mu}}
$$

is a hybrid submersion by Proposition 5.1. The hybrid manifold $\mathbf{M}_{\vec{\mu}}$ is called the reduced hybrid phase space.

\section{HybRid GEOMETRIC REDUCTION}

We now introduce the main theorem on reducing a hybrid symplectic manifold. We begin by reviewing the classic nonhybrid version of this theorem, originally proven by Marsden and Weinstien [10] (also see [1], [8], [9] for a more thorough account of classical reduction), followed by a statement of the hybrid version of this theorem.

Classical reduction. The starting point for classical reduction is a Hamiltonian $G$-space,

$$
(M, \omega, \Phi, J)
$$

where

- $(M, \omega)$ is a symplectic manifold,

- $\Phi: G \times M \rightarrow M$ is a symplectic action of a Lie group on $M$,

- $J$ is an $\mathrm{Ad}^{\star}$-equivariant momentum map for this action. 
With this notation, the classical reduction theorem reads:

Theorem 1: Let $(M, \omega, \Phi, J)$ be a Hamiltonian $G$-space and $\mu \in \mathfrak{g}^{\star}$ be a regular value of $J$. If the action of $G_{\mu}$ on $J^{-1}(\mu)$ is free and proper, then $M_{\mu}=J^{-1}(\mu) / G_{\mu}$ has a unique symplectic form $\omega_{\mu}$ with the property:

$$
\Omega^{2}\left(\pi_{\mu}\right)\left(\omega_{\mu}\right)=\Omega^{2}\left(i_{\mu}\right)(\omega),
$$

where $\pi_{\mu}: J^{-1}(\mu) \rightarrow M_{\mu}$ is the canonical projection and $i_{\mu}: J^{-1}(\mu) \rightarrow M$ is the inclusion.

The hybrid reduction theorem will nicely mirror and utilize this theorem. There also is an intriguing connection between the classical reduction theorem and hybrid symplectic manifolds; this theorem implies that the following hybrid manifold

$$
M \stackrel{i_{\mu}}{\longleftarrow} J^{-1}(\mu) \stackrel{\pi_{\mu}}{\longrightarrow} M_{\mu}
$$

is a hybrid symplectic manifold.

Hybrid Hamiltonian G-spaces. The necessary information needed in order to generalize this Theorem 1 is a hybrid Hamiltonian G-space, i.e., a tuple

$$
(\mathcal{M}, \mathbf{M}, \vec{\omega}, \vec{\Phi}, \vec{J})
$$

where

- $(\mathcal{M}, \mathbf{M}, \vec{\omega})$ is a hybrid symplectic manifold,

- $\vec{\Phi}: \mathbf{G} \times \mathbf{M} \dot{\rightarrow} \mathbf{M}$ is a symplectic hybrid action of a Lie group on $\mathbf{M}$,

- $\vec{J}$ is an $\overrightarrow{\mathrm{Ad}}^{\star}$-equivariant hybrid momentum map for this hybrid action.

For such a hybrid Hamiltonian G-space, we can reduce the dimensionality of $\mathbf{M}$ through hybrid reduction.

Theorem 2: Let $(\mathcal{M}, \mathbf{M}, \vec{\omega}, \vec{\Phi}, \vec{J})$ be a hybrid Hamiltonian $\mathbf{G}$-space. Assume $\vec{\mu} \in \mathfrak{g}^{\star}$ is a hybrid regular value of $\vec{J}$ and that the hybrid action of $\mathbf{G}_{\vec{\mu}}$ on $\mathbf{J}^{-1}(\vec{\mu})$ is free and proper. Then $\mathbf{M}_{\vec{\mu}}$ has a unique hybrid symplectic form $\vec{\omega}_{\vec{\mu}}$ with the property:

$$
\Omega_{*}^{2}\left(\vec{\pi}_{\vec{\mu}}\right)\left(\vec{\omega}_{\vec{\mu}}\right)=\Omega_{*}^{2}\left(\vec{i}_{\vec{\mu}}\right)(\vec{\omega}) .
$$

\section{HYBRID HAMILTONIAN REDUCTION}

The hybrid reduction theorem (Theorem 2) only gave conditions on when the phase space of a hybrid system can be reduced. In practice, we are interested in reducing the dynamics of a hybrid system. That is, we want to understand how to reduce hybrid Hamiltonians. This yields a method for reducing hybrid systems.

Dynamical systems. Consider the category of dynamical systems, Dyn, which has as

Objects: Dynamical systems, i.e., pairs $(M, X)$ where $M$ is a manifold and $X: M \rightarrow T M$ is a vector field on that manifold.

Morphisms: Smooth maps of manifolds $f: N \rightarrow$ $M$ such that there is a commuting diagram:

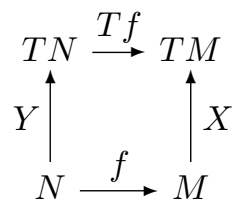

One of the motivations for considering this category is that it allows for a nice representation of trajectories of dynamical systems. That is, let Interval(Dyn) be the full subcategory of Dyn consisting of all dynamical systems of the form $(I, \mathrm{~d} / \mathrm{dt})$ with $I=\left[t_{0}, t_{1}\right],\left[t_{0}, t_{1}\right),\left\{t_{0}\right\}$ for $t_{0}, t_{1} \in \mathbb{R} \cup\{\infty\}$, $t_{0} \leq t_{1}$. A trajectory of a dynamical system $(M, X)$ is just a morphism in Dyn:

$$
c:(I, \mathrm{~d} / \mathrm{dt}) \rightarrow(M, X),
$$

where $(I, \mathrm{~d} / \mathrm{dt})$ is an object of Interval(Dyn); this implies that $\dot{c}(t)=X(c(t))$ as desired. The initial condition of such a trajectory is $c\left(t_{0}\right)$, with $t_{0}$ the left endpoint of $I$.

Classical Hamiltonian reduction. Before discussing how to reduce hybrid Hamiltonians, we review the classical Hamiltonian reduction theorem (cf. [1]). The setup for this theorem is a Hamiltonian $G$-space $(M, \omega, \Phi, J)$ satisfying the assumptions given in Theorem 1.

A Hamiltonian system is a tuple $(M, \omega, H)$, where $(M, \omega)$ is a symplectic manifold and $H: M \rightarrow \mathbb{R}$ is a Hamiltonian. From the Hamiltonian $H$, we obtain a vector field $X_{H}$ defined by $d(H)=\iota_{X_{H}}(\omega)$. That is, associated to the Hamiltonian system $(M, \omega, H)$ is a dynamical system $\left(M, X_{H}\right)$, or an object of Dyn.

A Hamiltonian $H: M \rightarrow \mathbb{R}$ is said to be $G$-invariant if for the action $\Phi: G \times M \rightarrow M$,

$$
H \circ \Phi(g,-)=H
$$

for all $g \in G$. From a $G$-invariant Hamiltonian, we obtain a Hamiltonian $H_{\mu}$ on $M_{\mu}$ defined by the requirement that:

$$
H \circ i_{\mu}=H_{\mu} \circ \pi_{\mu} \text {. }
$$

The end result is a reduced Hamiltonian system $\left(M_{\mu}, \omega_{\mu}, H_{\mu}\right)$, for which we have an associated dynamical system $\left(M_{\mu}, X_{H_{\mu}}\right)$.

The classical Hamiltonian reduction theorem relates trajectories of $\left(M, X_{H}\right)$ and trajectories of $\left(M_{\mu}, X_{H_{\mu}}\right)$. We state this result in a slightly different formulism, although it is equivalent to the standard result.

Theorem 3: Let $(M, \omega, \Phi, J)$ be a Hamiltonian $G$-space satisfying the assumptions given in Theorem 1 . If $H$ is a $G$-invariant Hamiltonian and $c:(I, \mathrm{~d} / \mathrm{dt}) \rightarrow\left(M, X_{H}\right)$ is a trajectory of $\left(M, X_{H}\right)$ with $c\left(t_{0}\right) \in J^{-1}(\mu)$, then

$$
c:(I, \mathrm{~d} / \mathrm{dt}) \rightarrow\left(J^{-1}(\mu), X_{H}\right),
$$

and there exists a trajectory $c_{\mu}:(I, \mathrm{~d} / \mathrm{dt}) \rightarrow\left(M_{\mu}, X_{H_{\mu}}\right)$ of $\left(M_{\mu}, X_{H_{\mu}}\right)$ defined by the factorization:

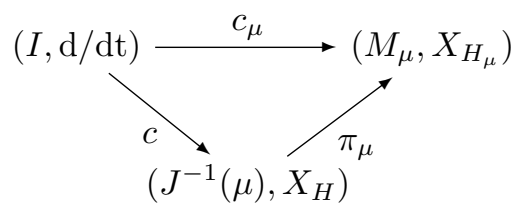

We now introduce the necessary groundwork needed in order to establish the hybrid analogue of Theorem 3.

Hybrid systems. A hybrid system is a tuple:

$$
(\mathcal{M}, \mathbf{M}, \mathbf{X}) \text {, }
$$


where

- $(\mathcal{M}, \mathbf{M})$ is a hybrid manifold,

- $\mathbf{X}=\left\{\mathbf{X}_{b}\right\}_{b \in \mathrm{V}(\mathcal{M})}$ is a collection of vector fields with $\mathbf{X}_{b}: \mathbf{M}_{b} \rightarrow T \mathbf{M}_{b}$ a vector field on $\mathbf{M}_{b}$, i.e., $\left(\mathbf{M}_{b}, \mathbf{X}_{b}\right)$ is an object of Dyn, for all $b \in \mathrm{V}(\mathcal{M})$.

This formulation of hybrid systems (which includes most "standard" definitions, see [7]) allows us to define the category of hybrid systems, HySys, which has as objects hybrid systems and as morphisms pairs:

$$
(\vec{F}, \vec{f}):(\mathcal{N}, \mathbf{N}, \mathbf{Y}) \rightarrow(\mathcal{M}, \mathbf{M}, \mathbf{X}),
$$

where $(\vec{F}, \vec{f}):(\mathcal{N}, \mathbf{N}) \rightarrow(\mathcal{M}, \mathbf{M})$ is a morphism in $\mathrm{Hy}($ Man) such that for all $b \in \mathrm{V}(\mathcal{N})$,

$$
\overrightarrow{f_{b}}:\left(\mathbf{N}_{b}, \mathbf{Y}_{b}\right) \rightarrow\left(\mathbf{M}_{\vec{F}(b)}, \mathbf{X}_{\vec{F}(b)}\right)
$$

is a morphism in Dyn.

The importance of considering the category of hybrid systems is that, in analogy with dynamical systems, one can define the interval subcategory of HySys, Interval(HySys); the details of this construction are, unfortunately, rather involved so we refer the reader to [2] (Chapter 2, page 62) for the specifics. The essential observation is that a trajectory of a hybrid system $(\mathcal{M}, \mathbf{M}, \mathbf{X})$ is a morphism in HySys:

$$
(\vec{C}, \vec{c}):(\mathcal{I}, \mathbf{I}, \mathbf{d} / \mathbf{d t}) \rightarrow(\mathcal{M}, \mathbf{M}, \mathbf{X}),
$$

where $(\mathcal{I}, \mathbf{I}, \mathbf{d} / \mathbf{d t})$ is an object of Interval(HySys). The initial condition of such a trajectory is $\left(\vec{C}(0), \vec{c}_{0}\left(\tau_{0}\right)\right)$ with $\vec{c}_{0}\left(\tau_{0}\right) \in \mathbf{M}_{\vec{C}(0)}$.

In the context of reduction, we are interested in studying hybrid systems obtained from hybrid Hamiltonians.

Definition 7.1: A hybrid Hamiltonian $\mathbf{H}$ on a hybrid manifold $(\mathcal{M}, \mathbf{M})$ is defined to be a set of maps:

$$
\mathbf{H}=\left\{\mathbf{H}_{b}: \mathbf{M}_{b} \rightarrow \mathbb{R}\right\}_{b \in \mathrm{V}(\mathcal{M})} .
$$

A hybrid Hamiltonian system is a tuple $(\mathcal{M}, \mathbf{M}, \vec{\omega}, \mathbf{H})$, where $(\mathcal{M}, \mathbf{M}, \vec{\omega})$ is a hybrid symplectic manifold and $\mathbf{H}$ is a hybrid Hamiltonian.

G-Invariant hybrid Hamiltonians. Let $(\mathcal{M}, \mathbf{G})$ be a hybrid Lie group acting on the hybrid manifold $(\mathcal{M}, \mathbf{M})$ through the hybrid action:

$$
\left(\overrightarrow{\mathrm{Id}}_{\mathcal{M}}, \vec{\Phi}\right):(\mathcal{M}, \mathbf{G} \times \mathbf{M}) \rightarrow(\mathcal{M}, \mathbf{M})
$$

A hybrid Hamiltonian $\mathbf{H}$ is said to be $\mathbf{G}$-invariant if

$$
\mathbf{H}_{b} \circ \vec{\Phi}_{b}(g,-)=\mathbf{H}_{b}
$$

for all $g \in \mathbf{G}_{b}$ and $b \in \mathrm{V}(\mathcal{M})$, i.e., $\mathbf{H}_{b}$ is $\mathbf{G}_{b}$-invariant for all $b \in \mathrm{V}(\mathcal{M})$.

Under the assumptions of Theorem 2, if $\mathbf{H}$ is a $\mathbf{G}$ invariant hybrid Hamiltonian on $(\mathcal{M}, \mathbf{M})$, then there is a hybrid Hamiltonian

$$
\mathbf{H}_{\vec{\mu}}=\left\{\left(\mathbf{H}_{\vec{\mu}}\right)_{b}:\left(\mathbf{M}_{\vec{\mu}}\right)_{b} \rightarrow \mathbb{R}\right\}_{b \in \mathrm{V}(\mathcal{M})}
$$

on $\mathbf{M}_{\vec{\mu}}$ defined by requiring that:

$$
\mathbf{H}_{b} \circ\left(\vec{i}_{\vec{\mu}}\right)_{b}=\left(\mathbf{H}_{\vec{\mu}}\right)_{b} \circ\left(\vec{\pi}_{\vec{\mu}}\right)_{b}
$$

for all $b \in \mathrm{V}(\mathcal{M})$. This defines a hybrid Hamiltonian system $\left(\mathcal{M}, \mathbf{M}_{\vec{\mu}}, \vec{\omega}_{\vec{\mu}}, \mathbf{H}_{\vec{\mu}}\right)$.

Hybrid Hamiltonian systems. From a hybrid Hamiltonian system $(\mathcal{M}, \mathbf{M}, \vec{\omega}, \mathbf{H})$ we obtain a hybrid system $\left(\mathcal{M}, \mathbf{M}, \mathbf{X}_{\mathbf{H}}\right)$, where $\mathbf{X}_{\mathbf{H}}$ is the collection of vector fields given by $\mathbf{X}_{\mathbf{H}}=\left\{\left(\mathbf{X}_{\mathbf{H}}\right)_{b}\right\}_{b \in \mathrm{V}(\mathcal{M})}$, with $\left(\mathbf{X}_{\mathbf{H}}\right)_{b}$ defined by the requirement that $d\left(\mathbf{H}_{b}\right)=\iota_{\left(\mathbf{X}_{\mathbf{H}}\right)_{b}}\left(\vec{\omega}_{b}\right)$. Similarly, we obtain a hybrid system $\left(\mathcal{M}, \mathbf{M}_{\vec{\mu}}, \mathbf{X}_{\mathbf{H}_{\vec{\mu}}}\right)$ from the hybrid Hamiltonian system $\left(\mathcal{M}, \mathbf{M}_{\vec{\mu}}, \vec{\omega}_{\vec{\mu}}, \mathbf{H}_{\vec{\mu}}\right)$.

We now demonstrate that the "dynamics" of $\mathbf{H}$ determine the corresponding "dynamics" of $\mathbf{H}_{\vec{\mu}}$ in the hybrid analogue to Theorem 3.

Theorem 4: Let $(\mathcal{M}, \mathbf{M}, \vec{\omega}, \vec{\Phi}, \vec{J})$ be a hybrid Hamiltonian $\mathbf{G}$-space satisfying the assumptions of Theorem 2. If $\mathbf{H}$ is a $\mathbf{G}$-invariant Hamiltonian and $(\vec{C}, \vec{c}):(\mathcal{I}, \mathbf{I}, \mathbf{d} / \mathbf{d t}) \rightarrow$ $\left(\mathcal{M}, \mathbf{M}, \mathbf{X}_{\mathbf{H}}\right)$ is a trajectory of $\left(\mathcal{M}, \mathbf{M}, \mathbf{X}_{\mathbf{H}}\right)$ with $\vec{c}_{0}\left(\tau_{0}\right) \in$ $\mathbf{J}^{-1}(\overrightarrow{\boldsymbol{\mu}})_{\vec{C}(0)}$, then

$$
(\vec{C}, \vec{c}):(\mathcal{I}, \mathbf{I}, \mathbf{d} / \mathbf{d t}) \rightarrow\left(\mathcal{M}, \mathbf{J}^{-\mathbf{1}}(\overrightarrow{\boldsymbol{\mu}}), \mathbf{X}_{\mathbf{H}}\right),
$$

and there exists a trajectory $\left(\vec{C}, \vec{c}_{\vec{\mu}}\right):(\mathcal{I}, \mathbf{I}, \mathbf{d} / \mathbf{d t}) \rightarrow$ $\left(\mathcal{M}, \mathbf{M}_{\vec{\mu}}, \mathbf{X}_{\mathbf{H}_{\vec{\mu}}}\right)$ of $\left(\mathcal{M}, \mathbf{M}_{\vec{\mu}}, \mathbf{X}_{\mathbf{H}_{\vec{\mu}}}\right)$ defined by the factorization:

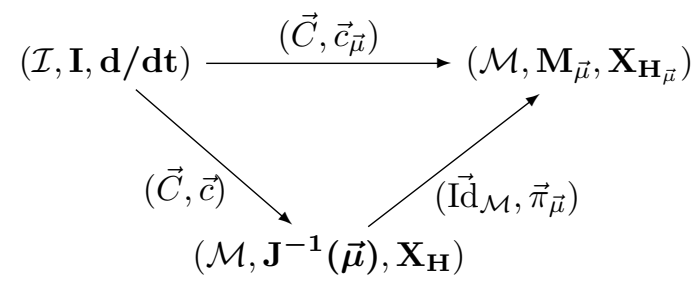

\section{ACKNOWLEDGEMENTS}

The first author is grateful to Paulo Tabuada for his assistance in refining the categorical framework for hybrid systems. The authors would also like to thank the anonymous reviewers for their insightful comments.

\section{REFERENCES}

[1] R. Abraham and J. E. Marsden, Foundations of Mechanics. Benjamin/Cummings Publishing Company, 1978.

[2] A. D. Ames, "A categorical theory of hybrid systems," Ph.D. dissertation, University of California, Berkeley, 2006.

[3] A. D. Ames and S. Sastry, "A homology theory for hybrid systems: Hybrid homology," in Hybrid Systems: Computation and Control, ser. Lecture Notes in Computer Science, M. Morari and L. Thiele, Eds., vol. 3414. Springer-Verlag, 2005, pp. 86-102.

[4] A. D. Ames, P. Tabuada, and S. Sastry, "On the stability of zeno equilibra," in Hybrid Systems: Computation and Control, ser. Lecture Notes in Computer Science, J. Hespanha and A. Tiwari, Eds., vol. 3927. Springer-Verlag, 2006, pp. 34-48.

[5] S. M. Lane, Categories for the Working Mathematician, 2nd ed., ser. Graduate Texts in Mathematics. Springer, 1998, vol. 5.

[6] J. M. Lee, Introduction to Smooth Manifolds, ser. Graduate Texts in Mathematics. Springer, 2003, vol. 218.

[7] J. Lygeros, K. H. Johansson, S. Simic, J. Zhang, and S. Sastry, "Dynamical properties of hybrid automata," IEEE Transactions on Automatic Control, vol. 48, pp. 2- 17, 2003.

[8] J. E. Marsden, Lectures on Mechanics, ser. London Mathematical Society Lecture Note Series. Cambridge University Press, 1992, vol. 174.

[9] J. E. Marsden and T. S. Ratiu, Introduction to Mechanics and Symmetry, ser. Texts in Applied Mathematics. Springer, 1999, vol. 17.

[10] J. E. Marsden and A. Weinstein, "Reduction of symplectic manifolds with symmetry," Rep. Math. Phys., vol. 5, pp. 121-130, 1974. 